\title{
Terminal Sliding Mode Control with Adaptive Law for Uncertain Nonlinear System
}

\author{
Zhanshan Zhao, ${ }^{1}$ Jing Zhang, ${ }^{2,3}$ Liankun Sun, ${ }^{1}$ and Dakun Zhang ${ }^{1}$ \\ ${ }^{1}$ School of Computer Science and Software Engineering, Tianjin Polytechnic University, Tianjin 300387, China \\ ${ }^{2}$ School of Textiles, Tianjin Polytechnic University, Tianjin 300387, China \\ ${ }^{3}$ Tianjin Vocational Institute, Tianjin 300410, China
}

Correspondence should be addressed to Jing Zhang; chufei726@163.com

Received 1 February 2015; Revised 16 April 2015; Accepted 20 April 2015

Academic Editor: Xinggang Yan

Copyright (C) 2015 Zhanshan Zhao et al. This is an open access article distributed under the Creative Commons Attribution License, which permits unrestricted use, distribution, and reproduction in any medium, provided the original work is properly cited.

\begin{abstract}
A novel nonsingular terminal sliding mode controller is proposed for a second-order system with unmodeled dynamics uncertainties and external disturbances. We need not achieve the knowledge for boundaries of uncertainties and external disturbances in advance. The adaptive control gains are obtained to estimate the uncertain parameters and external disturbances which are unknown but bounded. The closed loop system stability is ensured with robustness and adaptation by the Lyapunov stability theorem in finite time. An illustrative example of second-order nonlinear system with unmodeled dynamics and external disturbances is given to demonstrate the effectiveness of the presented scheme.
\end{abstract}

\section{Introduction}

Nonlinear control systems have been intensively studied in many control methods, including $H_{\infty}$ control, fault-tolerant control, robust passive control, neural networks, and fuzzy control [1-10]. Because sliding mode control (SMC) is well known for good robustness properties to system uncertainties and external disturbances [11-14], SMC has been widely studied as a powerful method to control nonlinear dynamic systems, such as stochastic systems [15-17] and nonlinear delay systems [18-22]. One of the conventional SMC characteristics is that the convergence time of the system states is usually asymptotical convergence from initial time to the equilibrium point, because we commonly choose the linear sliding mode manifold in spite of the conventional SMC claimed robustness.

A SMC based on finite time control, which can drive the system states to reach the equilibrium point in finite time, is studied in the literature of second-order sliding mode (SOSM) approach and terminal sliding mode (TSM) [2326]. It is worth noting that the switching manifold of SOSM is most linear switching manifold and the time derivative of the sliding variable needs to be obtained in advance.
We can compare linear switching manifolds based sliding modes with TSM. TSM has some good properties, for example, fast convergence to equilibrium point in finite time and high precision tracking performance. Because the conventional TSM control contains negative fractional powers which may cause singularity problem to occur, [24] designs a global nonsingular TSM controller to avoid the problem. However, the discontinuous nonsingular TSM control scheme can cause chattering phenomenon. In order to reduce the influence of chattering, an improved continuous TSM control is designed using the TSM switching manifold and reaching law [25]. Nonsingular TSMC has been widely developed because of fast convergence property in finite time without singularity problem, but the most nonsingular TSMC only deals with the disturbances which need to satisfy the matched condition. In order to effectively attenuate mismatched disturbances, [26] proposes a continuous TSMC approach to solve the mismatched disturbance problem based on a nonsingular TSMC technique and a finite time observer of disturbance. Although TSMC has been further utilized to extend the system with mismatched disturbance, the control design in [23-27] still has some problem to solve for uncertain nonlinear systems. For one thing, it is hard to acquire the 
knowledge of the boundaries of the system uncertainties in many practical cases.

In this paper, keeping the main advantages of the standard TSMC, we propose a novel adaptive nonsingular TSMC method for the control system with unmodeled uncertainties and external disturbances which are unknown but bounded. The designed control method is provided with fast convergence rate in finite time and strong robustness.

We organize the rest of the sections as follows. In Section 2, the dynamics of a class of uncertain nonlinear systems are introduced. In Section 3, the novel nonsingular TSM controller with adaptive law is developed to stabilize the uncertain nonlinear systems where we utilize the adaptive law to identify the uncertain boundaries. The novel adaptive nonsingular TSMC method can effectively provide strong robustness and fast convergence rate in finite time. Section 4 takes an example to validate that the proposed adaptive TSMC method has good performance for the uncertain nonlinear system. Finally, conclusions are presented in Section 5.

\section{Problem Statements}

Consider the following second-order uncertain nonlinear system which is depicted by

$$
\begin{aligned}
& \dot{x}_{1}=x_{2}, \\
& \dot{x}_{2}=f(x)+g(x) u+d(t),
\end{aligned}
$$

where $x=\left[x_{1}, x_{2}\right]^{T}$ is the system state vector, the functions $f(x), g(x)$ are uncertain but bounded smooth nonlinear functions, and $d(t)$ denotes the external disturbance and satisfies $|d(t)| \leq l_{g}$, where $l_{g}$ is the boundary and $l_{g}>0$.

The purpose of this paper is to stabilize the states of system (1) with the unmodeled dynamics and external disturbances by designing a control law $u(x)$ around the origin $x=[0,0]^{T}$.

A simple nonsingular TSM surface is proposed in order to overcome the singularity problem as follows [23]:

$$
s=x_{1}+\frac{1}{\beta} x_{2}^{q / p}
$$

From [23], the control law can be designed as follows:

$$
u=g^{-1}\left[-f-\beta \frac{q}{p} x_{2}^{2-p / q}-\left(l_{d}+\eta_{d}\right) \operatorname{sign} s\right]
$$

where $\eta_{d}$ is an arbitrary small positive constant.

The classical TSM and the nonsingular TSM controllers, including the continuous TSMC algorithm, can handle such problem in which the boundaries of the disturbances and parameter uncertainties are known. In this work, the objective is to design an adaptive gain TSM controller to make the states of uncertain nonlinear system converge to equilibrium point in finite time, in spite of the unmodeled uncertainties and external disturbances which are unknown but bounded. So, the gains of controller adapted the unknown boundaries.

\section{Nonsingular TSMC with Adaptive Law Design}

In this section, we propose a novel adaptive TSMC for uncertain nonlinear systems to get the stabilization of system states, which can assure robust convergence of the uncertain systems (1) in finite time under the unmodeled uncertainties and disturbances which are unknown but bounded. The main results of the paper are obtained as Theorem 4 based on [23], because these methods of [23] are only suitable for a special class of systems without unmodeled dynamics. In other words, the disturbance of the control input coefficient term $g(x)$ in system (1) is not considered which will be influenced to design control law.

Assumption 1. Solutions of the differential equation about NTSM surface (2) with discontinuous term of right-hand side are supposed in the sense of Filippov.

Assumption 2. The nonlinear functions $f(x), g(x)$ are partitioned into two parts. One is the nominal part; the other is uncertain bounded function. They can be expressed as follows:

$$
\begin{aligned}
& f(x)=f_{0}(x)+\Delta f(x), \\
& g(x)=g_{0}(x)+\Delta g(x) .
\end{aligned}
$$

The following inequalities are satisfied as

$$
\begin{aligned}
\left\|\Delta f(x)-\Delta g \cdot g_{0}^{-1} f_{0}+d(t)\right\| & \leq \rho(x, t), \\
\left\|\Delta g \cdot g_{0}^{-1}\right\| & \leq \alpha,
\end{aligned}
$$

where $|\rho(x, t)| \leq \rho$ is an unknown but bounded nonlinear function and parameter $0<\alpha<1$ is an unknown but bounded constant.

We apply the preliminary control feedback to system (1) as follows:

$$
u=g_{0}^{-1}\left[-f_{0}-\beta \frac{q}{p}\left|x_{2}\right|^{2-p / q} \cdot \operatorname{sign}\left(x_{2}\right)+w\right],
$$

where $\beta>0$ is an advanced given constant and $w$ is auxiliary control law which needs designing latter.

Lemma 3 (see [27]). The following system is considered:

$$
\begin{array}{r}
\dot{x}=f(x), \\
x(0)=x_{0}, \\
x \in R^{n}, f(0)=0,
\end{array}
$$

where $f(x): D \rightarrow R^{n}$ is continuous on an open neighborhood $D \subset R^{n}$. Suppose there is a continuous positive definite function $V(x): D \rightarrow R$, and there exist positive constants $\eta>0$, and $0<\gamma<1$, such that

$$
\dot{V}(x)+\eta V^{\gamma}(x) \leq 0 \text {. }
$$


Then, system (7) is locally finite time stable. Depending on the initial state $x(0)=x_{0}$, the settling time $T$ satisfies the following inequality as

$$
T \leq \frac{V^{1-\gamma}\left(x_{0}\right)}{\eta(1-\gamma)} .
$$

Particularly, when $D=R^{n}$ and $V(x)$ is also radially unbounded, the state of system (9) is globally finite time stable.

Thus, one obtains from system (1) using (6) the following:

$$
\begin{aligned}
\dot{x}_{1}= & x_{2}, \\
\dot{x}_{2}= & \left(1+\Delta g \cdot g_{0}^{-1}\right) w-\Delta g \cdot g_{0}^{-1} \cdot f_{0} \\
& -\left(1+\Delta g \cdot g_{0}^{-1}\right) \beta \frac{q}{p}\left|x_{2}\right|^{2-p / q} \cdot \operatorname{sign}\left(x_{2}\right) \\
& +\Delta f+d(t) .
\end{aligned}
$$

We take the time derivative of TSM surface s along the system trajectories using (2), (4), and (10) and can obtain the following:

$$
\begin{aligned}
\dot{s}= & \dot{x}_{1}+\frac{1}{\beta} \frac{p}{q} x_{2}^{p / q-1} \dot{x}_{2}=x_{2}+\frac{1}{\beta} \frac{p}{q} x_{2}^{p / q-1} \dot{x}_{2}=x_{2}+\frac{1}{\beta} \\
& \cdot \frac{p}{q} x_{2}^{p / q-1}(f(x)+g(x) u+d(t))=\frac{1}{\beta} \frac{p}{q} \\
& \cdot x_{2}^{p / q-1}\left[\left(1+\Delta g \cdot g_{0}^{-1}\right) w\right. \\
& -\Delta g \cdot g_{0}^{-1} \cdot f_{0}-\Delta g \cdot g_{0}^{-1} \beta \frac{q}{p}\left|x_{2}\right|^{2-p / q} \cdot \operatorname{sign}\left(x_{2}\right) \\
& +\Delta f+d(t)] .
\end{aligned}
$$

Theorem 4. Consider system (1) with unmodeled dynamics and external disturbances and suppose that Assumptions 1 and 2 are satisfied. The nonsingular TSM surface is selected as (2). The controller and adaptive law are designed as

$$
u=g_{0}^{-1}\left[-f_{0}-\beta \frac{q}{p}\left|x_{2}\right|^{2-p / q} \cdot \operatorname{sign}\left(x_{2}\right) \Theta \operatorname{sign} s\right],
$$

where the adaptive gain law $\Theta$ satisfies the following:

$$
\begin{aligned}
& \Theta=\zeta_{1}+\zeta_{2} \cdot\left|x_{2}\right|^{2-p / q}, \\
& \dot{\zeta}_{1}=\gamma_{1} \frac{1}{\beta} \frac{p}{q} x_{2}^{p / q-1} \cdot\|s\|, \\
& \dot{\zeta}_{2}=\gamma_{2} \frac{1}{\beta} \frac{p}{q} x_{2} \cdot\|s\|,
\end{aligned}
$$

where the positive constants $\bar{\zeta}_{i}(i=1,2)$ satisfy

$$
\begin{aligned}
& \bar{\zeta}_{1}>\frac{\rho}{1-\alpha} \\
& \bar{\zeta}_{2}>\frac{\alpha}{1-\alpha} \cdot \frac{\beta q}{p}
\end{aligned}
$$

The parameters $\beta, \gamma_{i}(i=1,2)$ are arbitrary positive constants in the adaptive gain law in (14), (15). $p$ and $q$ are positive odd integers and satisfy $1<p / q<2$, which ensures converging to zero with respect to NTSM surface s in finite time.

Proof. The following Lyapunov function is chosen as follows:

$$
V=\frac{1}{2} s^{2}+\sum_{i=1}^{2} \frac{1}{2 \gamma_{i}}\left(\zeta_{i}-\bar{\zeta}_{i}\right)^{2}
$$

The time derivative of Lyapunov function (17) with condition (16) is calculated as

$$
\dot{V}=s \dot{s}+\sum_{i=1}^{2} \frac{1}{\gamma_{i}}\left(\zeta_{i}-\bar{\zeta}_{i}\right) \cdot \dot{\zeta}_{i}
$$

The proof is divided into two cases.

Case $1\left(x_{2} \neq 0\right)$. We take the derivative of the TSM surface (2) along the system dynamics (1) under the condition of controller (12) and the adaptive gain law (13)-(15).

So, we can obtain from (11)

$$
\begin{aligned}
s \dot{s} & =\frac{1}{\beta} \frac{p}{q} \\
& \cdot x_{2}^{p / q-1}\left\{\left(1+\Delta g \cdot g_{0}^{-1}\right)\left(\zeta_{1}+\zeta_{2} \cdot\left|x_{2}\right|^{2-p / q}\right) \text { sign } s\right. \\
& +\left[\Delta f-\Delta g \cdot g_{0}^{-1} \cdot f_{0}+d(t)\right]-\Delta g \\
& \left.\cdot g_{0}^{-1} \beta \frac{q}{p}\left|x_{2}\right|^{2-p / q} \cdot \operatorname{sign}\left(x_{2}\right)\right\}=\frac{1}{\beta} \frac{p}{q} \\
& \cdot x_{2}^{p / q-1}\left\{-\left(\zeta_{1}+\zeta_{2} \cdot\left|x_{2}\right|^{2-p / q}\right)\right. \\
& \cdot\|s\|-\Delta g \cdot g_{0}^{-1} \cdot\left(\zeta_{1}+\zeta_{2} \cdot\left|x_{2}\right|^{2-p / q}\right) \cdot\|s\| \\
& +\left[\Delta f-\Delta g \cdot g_{0}^{-1} \cdot f_{0}+d(t)\right] \cdot s-\Delta g \\
& \left.\cdot g_{0}^{-1} \beta \frac{q}{p}\left|x_{2}\right|^{2-p / q} \cdot \operatorname{sign}\left(x_{2}\right) \cdot s\right\} \leq \frac{1}{\beta} \frac{p}{q} \\
& \left.+\alpha \beta \frac{q}{p}\left|x_{2}\right|^{2-p / q} \cdot\|s\|\right] \cdot \\
& \cdot\|s\|+\alpha-\left(\zeta_{1}+\zeta_{1}+\zeta_{2} \cdot\left|x_{2}\right|^{2-p / q}\right) \\
& \left.+\zeta_{2} \cdot\left|x_{2}\right|^{2-p / q}\right) \cdot\|s\|+\rho \cdot\|s\|
\end{aligned}
$$

So,

$$
\begin{aligned}
\dot{V} & \leq \frac{1}{\beta} \frac{p}{q} x_{2}^{p / q-1}\left[-\left(\zeta_{1}+\zeta_{2} \cdot\left|x_{2}\right|^{2-p / q}\right) \cdot\|s\|+\alpha \cdot\left(\zeta_{1}\right.\right. \\
& \left.+\zeta_{2} \cdot\left|x_{2}\right|^{2-p / q}\right) \cdot\|s\|+\rho \cdot\|s\|+\alpha \beta \frac{q}{p}\left|x_{2}\right|^{2-p / q}
\end{aligned}
$$




$$
\begin{aligned}
& \cdot\|s\|]+\sum_{i=1}^{2} \frac{1}{\gamma_{i}}\left(\zeta_{i}-\bar{\zeta}_{i}\right) \cdot \dot{\zeta}_{i} \leq \frac{1}{\beta} \frac{p}{q} x_{2}^{p / q-1}\left[-\left(\zeta_{1}\right.\right. \\
& \left.+\zeta_{2} \cdot\left|x_{2}\right|^{2-p / q}\right) \cdot\|s\|+\alpha \cdot\left(\zeta_{1}+\zeta_{2} \cdot\left|x_{2}\right|^{2-p / q}\right) \\
& \left.\cdot\|s\|+\rho \cdot\|s\|+\alpha \frac{k}{\lambda}\left|x_{2}\right|^{2-p / q} \cdot\|s\|\right]+\left(\zeta_{1}-\bar{\zeta}_{1}\right) \cdot \frac{1}{\beta} \\
& \cdot \frac{p}{q} x_{2}^{p / q-1} \cdot\|s\|+\left(\zeta_{2}-\bar{\zeta}_{2}\right) \cdot \frac{1}{\beta} \frac{p}{q} x_{2}^{p / q-1} \cdot\left|x_{2}\right|^{2-p / q} \\
& \cdot\|s\| \leq \frac{1}{\beta} \frac{p}{q} x_{2}^{p / q-1}\left[-\bar{\zeta}_{1} \cdot\|s\|-\bar{\zeta}_{2} \cdot\left|x_{2}\right|^{2-p / q} \cdot\|s\|\right. \\
& +\alpha \cdot\left(\zeta_{1}+\zeta_{2} \cdot\left|x_{2}\right|^{2-p / q}\right) \cdot\|s\|+\rho \cdot\|s\|+\alpha \beta \\
& \left.\cdot \frac{q}{p}\left|x_{2}\right|^{2-p / q} \cdot\|s\|\right] \leq-\frac{1}{\beta} \frac{p}{q} x_{2}^{p / q-1}\left\{\left[(1-\alpha) \bar{\zeta}_{1}\right.\right. \\
& \left.-\rho+(1-\alpha) \bar{\zeta}_{2} \cdot\left|x_{2}\right|^{2-p / q}-\alpha \beta \frac{q}{p} \cdot\left|x_{2}\right|^{2-p / q}\right] \\
& \cdot\|s\|+\alpha\|s\| \cdot\left\|\zeta_{1}-\bar{\zeta}_{1}\right\|+\alpha\|s\| \cdot\left|x_{2}\right|^{2-p / q} \cdot \| \zeta_{2} \\
& \left.-\bar{\zeta}_{2} \|\right\} \leq-\frac{1}{\beta} \frac{p}{q} x_{2}^{p / q-1}\left\{\sqrt { 2 } \left[(1-\alpha) \bar{\zeta}_{1}-\rho\right.\right. \\
& \left.+(1-\alpha) \bar{\zeta}_{2} \cdot\left|x_{2}\right|^{2-p / q}-\alpha \beta \frac{q}{p} \cdot\left|x_{2}\right|^{2-p / q}\right] \cdot \frac{\|s\|}{\sqrt{2}} \\
& +\sqrt{2 \gamma_{1}} \alpha\|s\| \cdot \frac{\left\|\zeta_{1}-\bar{\zeta}_{1}\right\|}{\sqrt{2 \gamma_{1}}}+\sqrt{2 \gamma_{2}} \alpha\|s\| \cdot\left|x_{2}\right|^{2-p / q} \\
& \left.\frac{\left\|\zeta_{2}-\bar{\zeta}_{2}\right\|}{\sqrt{2 \gamma_{2}}}\right\} \leq-\eta_{0} \cdot\left(\frac{\|s\|}{\sqrt{2}}+\frac{\left\|\zeta_{1}-\bar{\zeta}_{1}\right\|}{\sqrt{2 \gamma_{1}}}\right. \\
& \left.+\frac{\left\|\zeta_{2}-\bar{\zeta}_{2}\right\|}{\sqrt{2 \gamma_{2}}}\right)
\end{aligned}
$$

where

$$
\eta_{0}=\frac{1}{\beta} \frac{p}{q} x_{2}^{p / q-1} \cdot \min \left(r_{0}, r_{1}, r_{2}\right) .
$$

Order

$$
\begin{aligned}
r_{0} & =\sqrt{2}\left[(1-\alpha) \bar{\zeta}_{1}-\rho+(1-\alpha) \bar{\zeta}_{2} \cdot\left|x_{2}\right|^{2-p / q}\right. \\
& \left.-\alpha \beta \frac{q}{p} \cdot\left|x_{2}\right|^{2-p / q}\right] \\
r_{1} & =\sqrt{2 \gamma_{1}} \alpha\|s\| \\
r_{2} & =\sqrt{2 \gamma_{2}} \alpha\|s\| \cdot\left|x_{2}\right|^{2-p / q} .
\end{aligned}
$$

Because $q$ is positive odd integer and satisfies inequality (16), we can get $r_{0}>0$ and $\eta_{0}>0$.
We apply a well-known inequality for (20) as follows:

$$
\left(x^{2}+y^{2}+z^{2}\right)^{1 / 2} \leq|x|+|y|+|z|
$$

One obtains

$$
\dot{V}<-\eta_{0} V^{1 / 2}
$$

According to Lemma 3, we can obtain system (1) is converged to NTSM surface $s=0$ in finite time which meets $t_{F} \leq$ $V^{1 / 2}\left(x_{0}\right) / 2 \eta_{0}$. Thus, system state trajectories are evolved on the surface $s=0$ in finite time and remain there with the unmodeled uncertainties and external disturbance in $x_{2} \neq 0$.

Case $2\left(x_{2}=0\right)$. If $x_{2}=0$, we know that the NTSM surface in (2) becomes as follows:

$$
s=x_{1}
$$

where is induced $s \dot{s}=x_{1} x_{2}=0$, so, $x_{2}=0$ is a possible attractor. Then, we should discuss the additional case which is necessary to be considered. In this extreme case, the control law (12) is represented as follows:

$$
u=g_{0}^{-1}\left[-f_{0}-\Theta \operatorname{sign} s\right]
$$

We can substitute the control law (12) into system (1). One obtains

$$
\begin{aligned}
\dot{x}_{2}= & -\Theta\left(1+\Delta g \cdot g_{0}^{-1}\right) \operatorname{sign} s+\Delta f(x)-\Delta g \cdot g_{0}^{-1} f_{0} \\
& +d(t) .
\end{aligned}
$$

In the sequel, we can prove the inequality $x_{1} \cdot \dot{x}_{2}<0$ in order to confirm that $x_{2}=0$ is not an attractor in the phase plane. $x_{1}$ is multiplied by (27). One yields

$$
\begin{aligned}
& x_{1} \cdot \dot{x}_{2}=s \cdot \dot{x}_{2}=s \cdot\left[-\Theta\left(1+\Delta g \cdot g_{0}^{-1}\right) \text { sign } s\right. \\
& \left.\quad+\Delta f(x)-\Delta g \cdot g_{0}^{-1} f_{0}+d(t)\right] \leq-\Theta\|s\|+(1-\alpha) \\
& \quad \cdot \Theta\|s\|+\rho(x)\|s\| \leq-\eta\|s\|<0 .
\end{aligned}
$$

Through inequality (23) which is valid, we can get $x_{2}=0$ is not an attractor. The points are obtained as follows:

(a) When $x_{1}<0$ and $x_{1}>0$, we can, respectively, get $\dot{x}_{2}>0$ and $\dot{x}_{2}<0$ from (27).

(b) There exist a neighborhood of $x_{2}=0$ and a small positive number $\varepsilon>0$. When $\left|x_{2}\right|<\varepsilon$, we can, respectively, obtain $\dot{x}_{2}>0$ for $x_{1}<0$ and $\dot{x}_{2}<0$ for $x_{1}>0$.

(c) If $x_{1}<0$, the trajectory crosses from the boundary of the neighborhood $x_{2}=-\varepsilon$ to $x_{2}=\varepsilon$; else if $x_{1}>0$ the trajectory crosses from $x_{2}=\varepsilon$ to $x_{2}=-\varepsilon$. So, we can see that $x_{2}=0$ is not an attractor.

(d) When $\left|x_{2}\right|>\varepsilon$, we can easily conclude that the NTSM surface is reached in finite time because of $x_{1} \cdot \dot{x}_{2}<0$. 
From the above analysis, we can conclude that the NTSM surface $s=0$ which is defined in (2) can be reached from anywhere in the finite time. The requirements depicted in this theorem are satisfied as design of control law (12)-(15).

Remark. In order to reduce the effect of chattering, we approximately replace $\operatorname{sign}(s)$ with the function $\tanh (s / \varepsilon)$ in controller (12), where $\varepsilon>0$ is a sufficient small constant. We apply the modification in design of controller (14) for the later numerical simulation studies.

\section{Numerical Simulations}

To illustrate the effectiveness of the above given scheme, we consider the following design example to verify TSMC with adaptive law in this section. A second-order nonlinear system with unmodeled dynamics and external disturbances is considered:

$$
\begin{aligned}
\dot{x}_{1}= & x_{2}, \\
\dot{x}_{2}= & \cos x_{1}+0.2 \sin \left(x_{2} \cdot t\right) \\
& +\left(x_{1}^{2}+1+0.1 \cos (5 \pi t)\right) u \\
& +0.02 \sin (2 \pi t),
\end{aligned}
$$

where $f_{0}(x)=\cos x_{1}, \Delta f=0.2 \sin \left(x_{2} \cdot t\right), g_{0}(x)=x_{1}^{2}+1$, $\Delta g=0.1 \cos (5 \pi t), d(t)=0.02 \sin (2 \pi t)$, and the initial state of system $(29)$ is $[0.5,0.1]$. We apply the terminal sliding mode control with adaptive law (9)-(12) and choose the related parameters as follows: $\beta=5, q=3, p=5, \gamma_{1}=3$, and $\gamma_{2}=5$. Figure 1 shows the states of system (29). The control input signals for system (29) based on controller (12) are demonstrated in Figure 2. The nonsingular TSM surface is designed in Figure 3. Some valuable facts are obviously seen; for example, the problem of singularity does not occur at all times of NTSM control and the states of system (29) can converge to zero in finite time in Figures 1-3, 6, and 7. Figures 1-3, 6, and 7 display that the controller design (12)(15) is effective in spite of unknown uncertain boundaries. The NTSM surface rapidly approaches zero in Figure 3 . The estimation value responses of control gain parameters $\gamma_{1}, \gamma_{2}$ are, respectively, shown in Figures 4 and 5. Figures 4 and 5 show that the control gain parameters tend to a constant value. By comparing Figures 1 and 2 with Figures 6 and 7 , we can obtain that the chattering effect is reduced by the modification in design of controller (12). The results of simulations reveal that the proposed terminal sliding mode control with adaptive law is indeed effective in practice in spite of the existence of second-order system unmodeled dynamics and external disturbances.

\section{Conclusions}

This work proposes TSMC based on adaptive law method for second-order nonlinear system with unmodeled dynamics where external disturbances and the boundaries of the nonlinear dynamics and external disturbances are not required

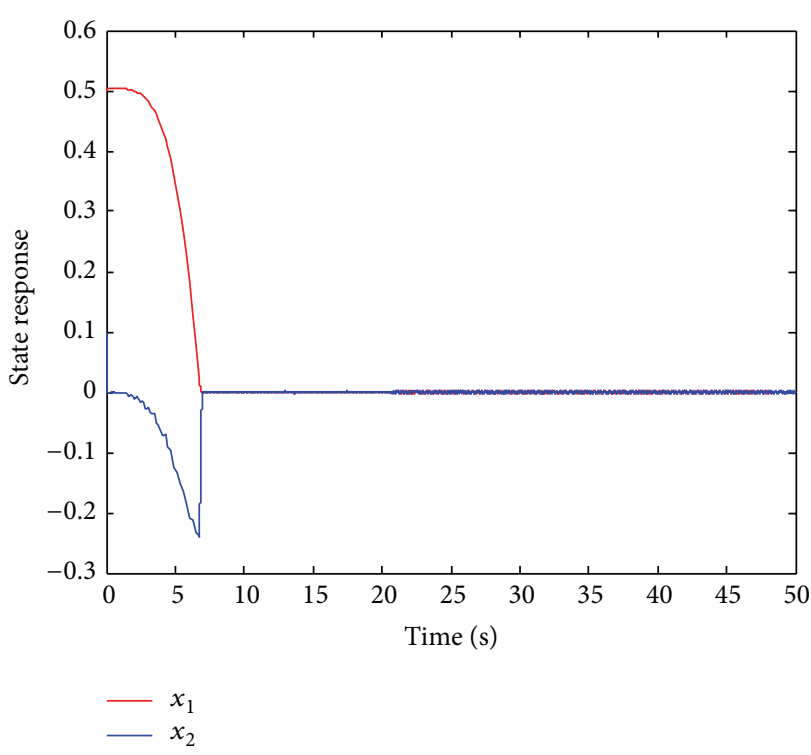

FIGURE 1: The states of system (29) based on control (12).

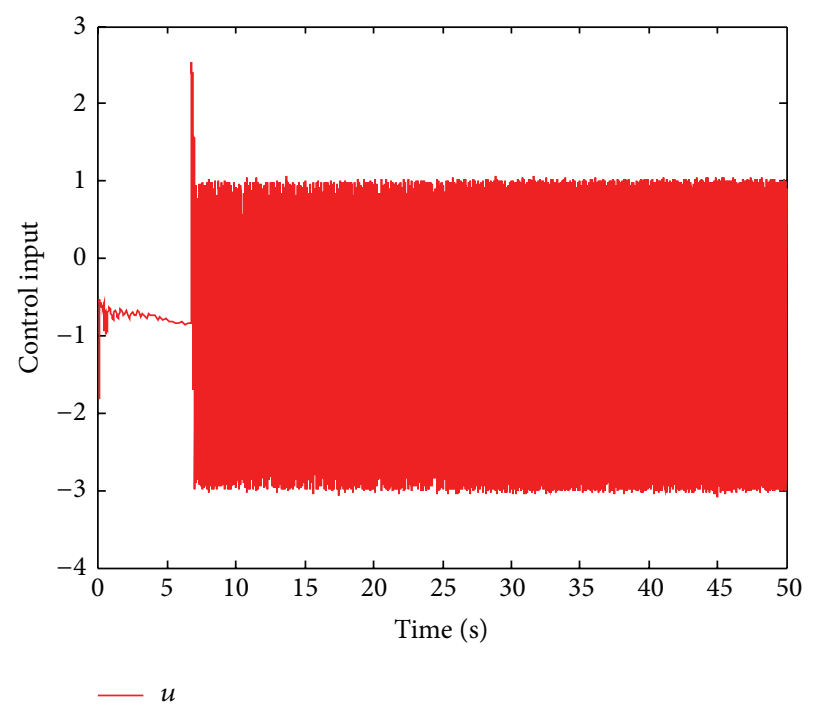

FIgURE 2: Control input signal of (12).

in advance. The proposed terminal sliding mode control with adaptive law method is proved to guarantee robustness and adaptation by the Lyapunov stability method in finite time. The control gain parameters are automatically adjusted by adaptive law and the nonsingular TSM controller effectively prevents the singularity problem from occurring. A numerical simulation is demonstrated to verify the effectiveness of robustness and adaptation using the presented nonsingular TSMC with adaptive law method. The further research are the extension of this paper to stochastic systems methods based on fuzzy control and adaptive neural network control which will bring big challenges for terminal sliding mode control design [28-30]. 


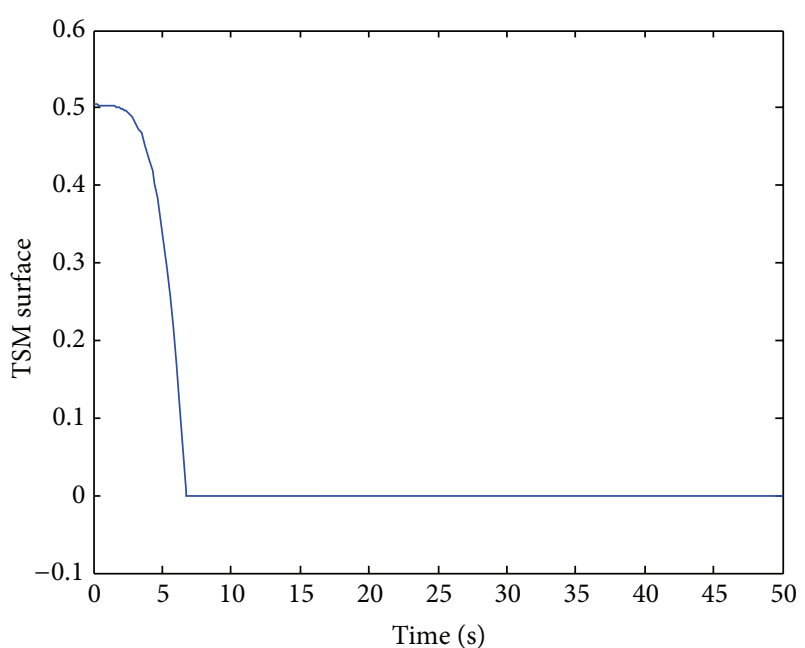

$-s$

FIgURE 3: The nonsingular TSM surface.

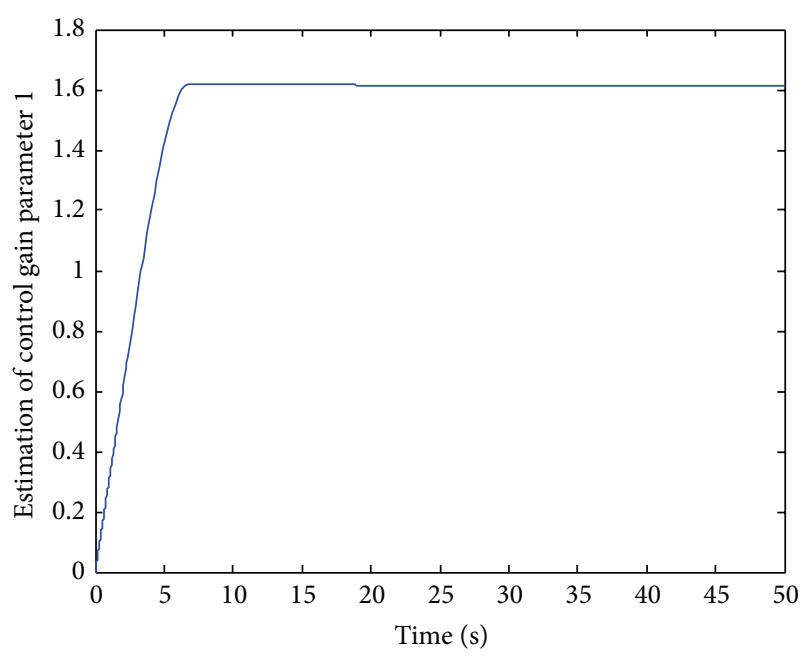

Figure 4: Control gain parameter $\zeta_{1}$.

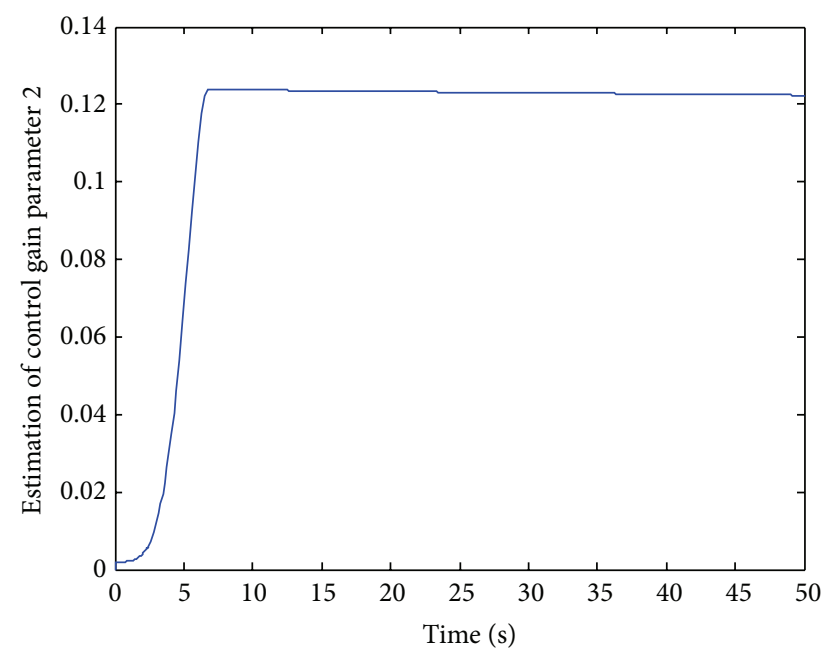

Figure 5: Control gain parameter $\zeta_{2}$.

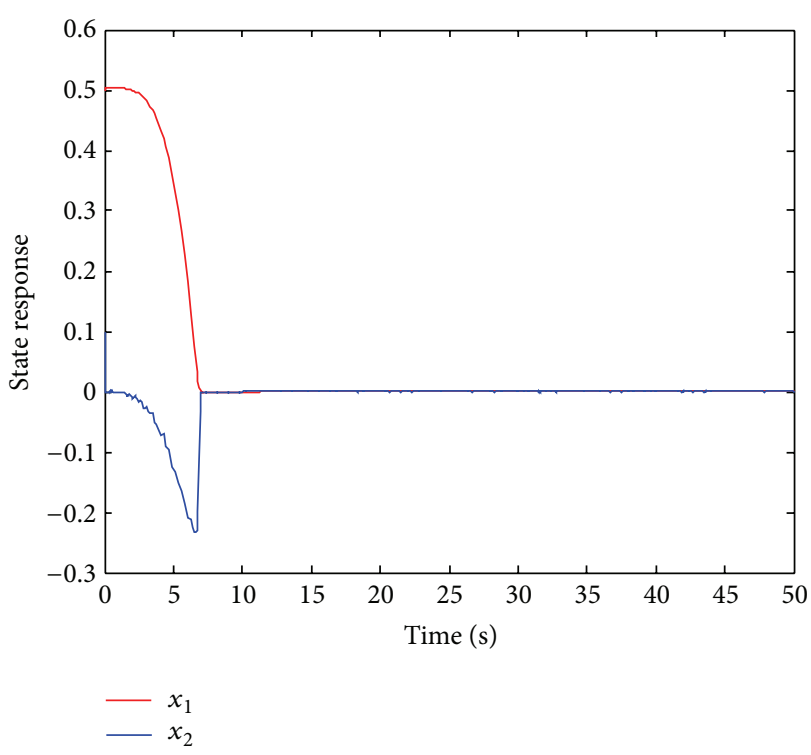

FIGURE 6: The states of system (29) based on the modified control (12).

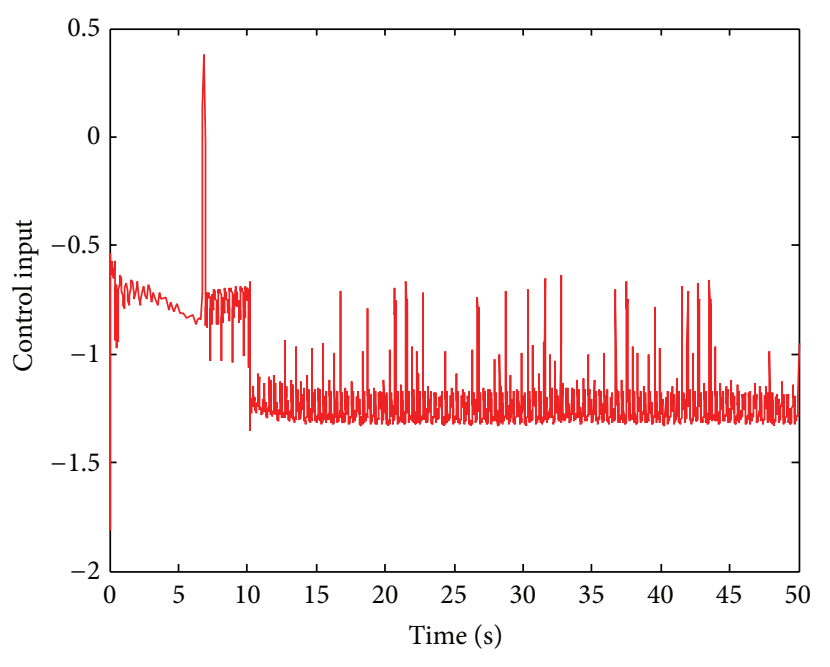

FIGURE 7: The modified control input signal of (12).

\section{Conflict of Interests}

The authors declare that there is no conflict of interests regarding the publication of this paper.

\section{Acknowledgments}

This work is partially supported by National Natural Science Foundation of China under Grant nos. 61503280, 61403278, and 61272006 . The authors also gratefully acknowledge the anonymous reviewers for their valuable comments. 


\section{References}

[1] L. Li, Q. L. Zhang, and B. Y. Zhu, " $H_{\infty}$ fuzzy control for nonlinear time-delay singular Markovian jump systems with partly unknown transition rates," Fuzzy Sets and Systems, vol. 254, no. 1, pp. 106-125, 2014.

[2] J. Zhu, Q. L. Zhang, and Z. Yuan, "Delay-dependent passivity criterion for discrete-time delayed standard neural network model," Neurocomputing, vol. 73, no. 7-9, pp. 1384-1393, 2010.

[3] J. Zhu, Q. Zhang, and C. Yang, "Delay-dependent robust stability for Hopfield neural networks of neutral-type," Neurocomputing, vol. 72, no. 10-12, pp. 2609-2617, 2009.

[4] Q. Li, Q. L. Zhang, N. Yi, and Y. H. Yuan, "Robust passive control for uncertain time-delay singular systems," IEEE Transactions on Circuits and Systems, vol. 56, no. 3, pp. 653-663, 2009.

[5] Z. P. Du, Q. L. Zhang, and Y. Li, "Delay-dependent robust $H_{\infty}$ control for uncertain singular systems with multiple state delays," IET Control Theory \& Applications, vol. 3, no. 6, pp. 731740, 2009.

[6] Y. Ma, N. Gu, and Q. L. Zhang, "Non-fragile robust $H_{\infty}$ control for uncertain discrete-time singular systems with time-varying delays," Journal of the Franklin Institute: Engineering and Applied Mathematics, vol. 351, no. 6, pp. 3163-3181, 2014.

[7] H. Y. Li, X. J. Jing, H. K. Lam, and P. Shi, "Fuzzy sampleddata control for uncertain vehicle suspension systems," IEEE Transactions on Cybernetics, vol. 44, no. 7, pp. 1111-1126, 2014.

[8] H. Y. Li, X. J. Jing, and H. R. Karimi, "Output-feedback-based $H_{\infty}$ control for vehicle suspension systems with control delay," IEEE Transactions on Industrial Electronics, vol. 61, no. 1, pp. 436-446, 2014.

[9] H. Y. Li, H. J. Gao, P. Shi, and X. D. Zhao, "Fault-tolerant control of Markovian jump stochastic systems via the augmented sliding mode observer approach," Automatica, vol. 50, no. 7, pp. 1825-1834, 2014.

[10] H. Y. Li, X. J. Sun, P. Shi, and H. K. Lam, "Control design of interval type-2 fuzzy systems with actuator fault: sampled-data control approach," Information Sciences, vol. 302, pp. 1-13, 2015.

[11] A. Rahman, S. K. Spurgeon, and X. G. Yan, "Estimation and control of non-linear variables in a continuous fermentation process using sliding mode techniques," Transactions of the Institute of Measurement and Control, vol. 34, no. 7, pp. 769-779, 2012.

[12] H. Y. Li, J. Yu, C. Hilton, and H. Liu, "Adaptive sliding-mode control for nonlinear active suspension vehicle systems using $\mathrm{T}$ S fuzzy approach," IEEE Transactions on Industrial Electronics, vol. 60, no. 8, pp. 3328-3338, 2013.

[13] L. Wu and H. Gao, "Sliding mode control of two-dimensional systems in Roesser model," IET Control Theory \& Applications, vol. 2, no. 4, pp. 352-364, 2008.

[14] L. Wu, H. Gao, and C. Wang, "Quasi sliding mode control of differential linear repetitive processes with unknown input disturbance," IEEE Transactions on Industrial Electronics, vol. 58, no. 7, pp. 3059-3068, 2011.

[15] L. Wu, W. X. Zheng, and H. Gao, "Dissipativity-based sliding mode control of switched stochastic systems," IEEE Transactions on Automatic Control, vol. 58, no. 3, pp. 785-791, 2013.

[16] L. Wu, P. Shi, and H. Gao, "State estimation and slidingmode control of Markovian jump singular systems," IEEE Transactions on Automatic Control, vol. 55, no. 5, pp. 1213-1219, 2010.
[17] L. Wu and D. W. Ho, "Sliding mode control of singular stochastic hybrid systems," Automatica, vol. 46, no. 4, pp. 779783, 2010.

[18] X.-G. Yan, S. K. Spurgeon, and C. Edwards, "State and parameter estimation for nonlinear delay systems using sliding mode techniques," IEEE Transactions on Automatic Control, vol. 58, no. 4, pp. 1023-1029, 2013.

[19] X.-G. Yan, S. K. Spurgeon, and C. Edwards, "Global decentralised static output feedback sliding-mode control for interconnected time-delay systems," IET Control Theory and Applications, vol. 6, no. 2, pp. 192-202, 2012.

[20] X.-G. Yan and S. K. Spurgeon, "Decentralised delay-dependent static output feedback variable structure control," Journal of the Franklin Institute, vol. 351, no. 4, pp. 2033-2047, 2014.

[21] X.-G. Yan and S. K. Spurgeon, "Memoryless decentralised static output feedback variable structure control synthesis for time varying delay interconnected systems," in Proceedings of the 12th International Workshop on Variable Structure Systems (VSS '12), pp. 379-384, January 2012.

[22] X.-G. Yan, S. K. Spurgeon, and Y. Orlov, "Sliding mode observer based control for a class of nonlinear time delay systems with delayed uncertainties," in Proceedings of the IEEE Conference on Decision and Control, pp. 7611-7616, December 2011.

[23] Y. Feng, X. Yu, and F. Han, "On nonsingular terminal slidingmode control of nonlinear systems," Automatica, vol. 49, no. 6, pp. 1715-1722, 2013.

[24] Y. Feng, X. Yu, and Z. Man, "Non-singular terminal sliding mode control of rigid manipulators," Automatica, vol. 38, no. 12, pp. 2159-2167, 2002.

[25] S. Yu, X. Yu, B. Shirinzadeh, and Z. Man, "Continuous finitetime control for robotic manipulators with terminal sliding mode," Automatica, vol. 41, no. 11, pp. 1957-1964, 2005.

[26] J. Yang, S. Li, J. Su, and X. Yu, "Continuous nonsingular terminal sliding mode control for systems with mismatched disturbances," Automatica, vol. 49, no. 7, pp. 2287-2291, 2013.

[27] S. P. Bhat and D. S. Bernstein, "Finite-time stability of continuous autonomous systems," SIAM Journal on Control and Optimization, vol. 38, no. 3, pp. 751-766, 2000.

[28] T. Wang, H. Gao, and J. Qiu, "A combined adaptive neural network and nonlinear model predictive control for multirate networked industrial process control," IEEE Transactions on Neural Networks and Learning Systems, no. 99, 2015.

[29] H. Y. Li, C. W. Wu, P. Shi, and Y. B. Gao, "Control of nonlinear networked systems with packet dropouts: interval type-2 fuzzy model-based approach," IEEE Transactions on Cybernetics, 2014.

[30] H. Y. Li, C. W. Wu, L. G. Wu, H. K. Lam, and Y. B. Gao, "Filtering of interval type-2 fuzzy systems with intermittent measurements," IEEE Transactions on Cybernetics, 2015. 


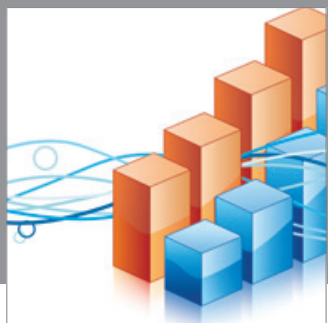

Advances in

Operations Research

mansans

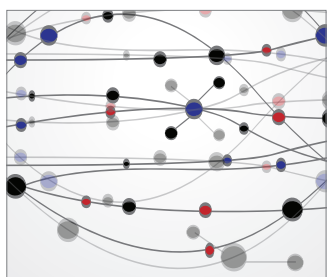

The Scientific World Journal
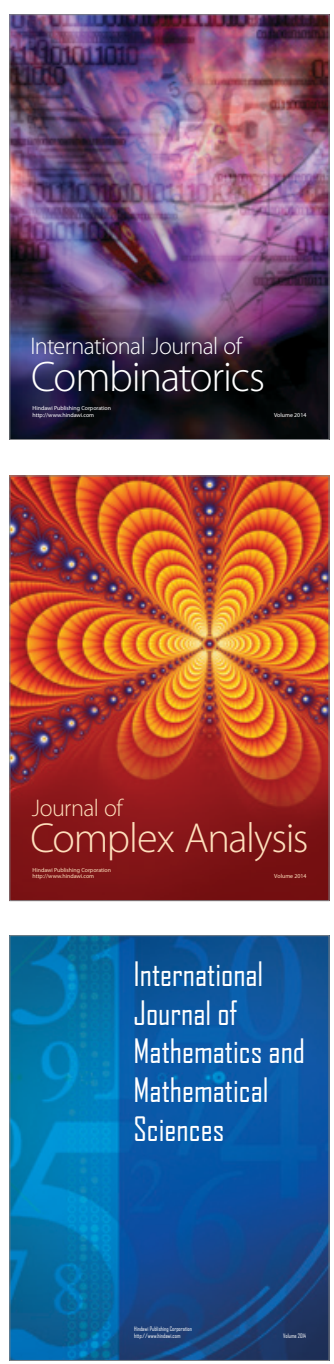
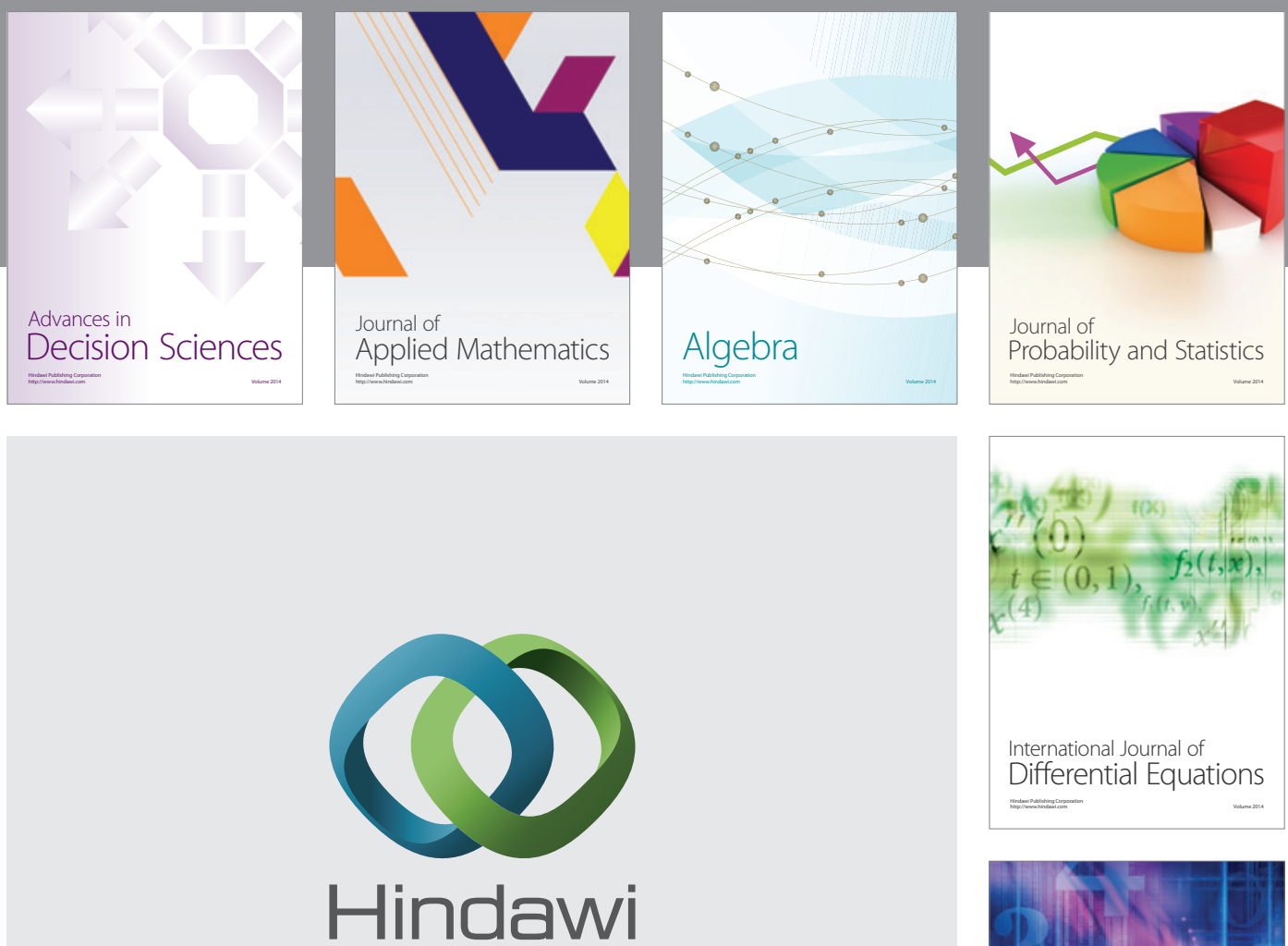

Submit your manuscripts at http://www.hindawi.com
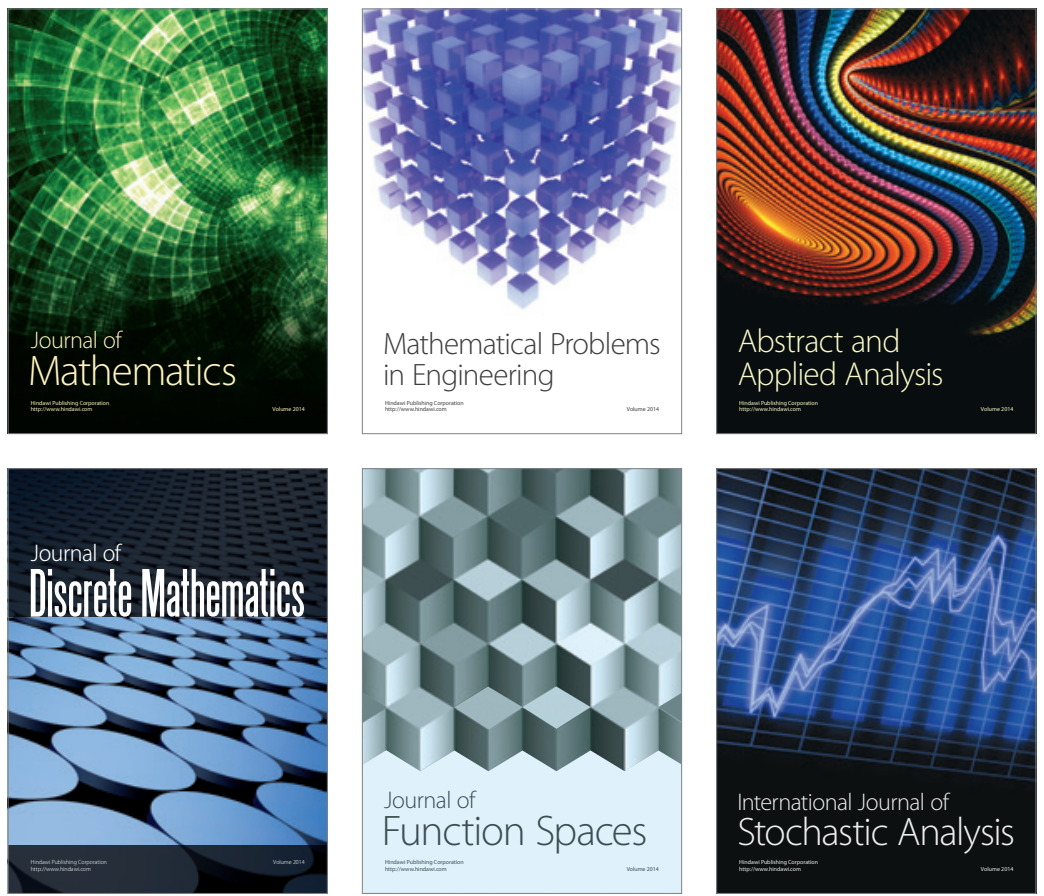

Journal of

Function Spaces

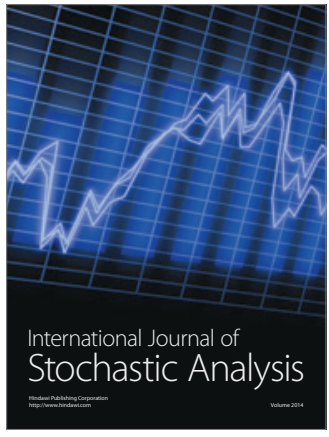

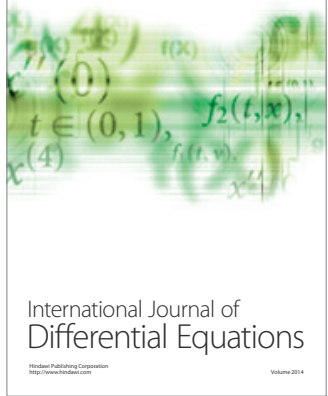
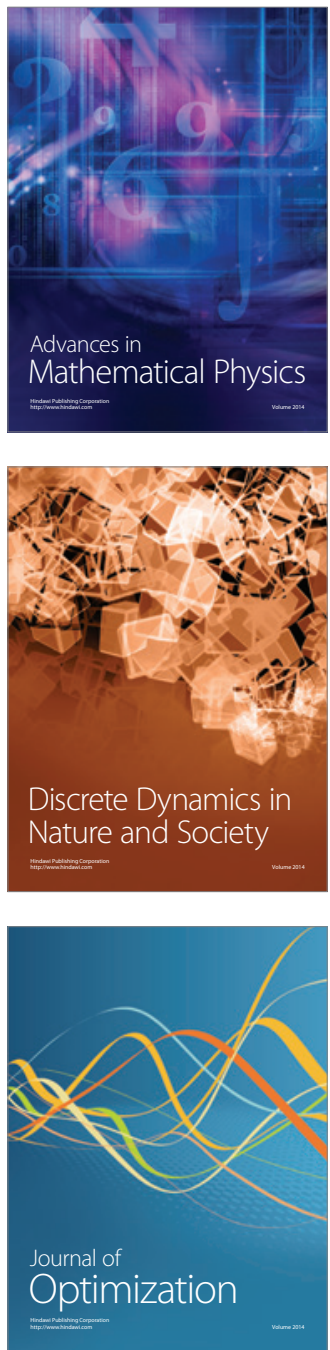